
\title{
ACREDITACIÓN DE ALTA CALIDAD DEL PROGRAMA DE MERCADEO Y PUBLICIDAD DE LA INSTITUCIÓN UNIVERSITARIA POLITÉCNICO GRANCOLOMBIANO
}

\section{Self-assessment and Renewal of the High Quality Certification Given to the Marketing and Advertising Program of Politécnico Grancolombiano University Institution}

\section{Autoavaliação e renovação da acreditação de alta qualidade do programa de Marketing e Publicidade da Instituição Universitária Politécnico Grancolombiano}

\section{es}

\section{RESUMEN}

El presente artículo, resultado de investigación, pretende registrar el proceso de autoevaluación orientado a la renovación de la acreditación de alta calidad del programa de Mercadeo y Publicidad de la Institución Universitaria Politécnico Grancolombiano en concordancia con los lineamientos del Consejo Nacional de Acreditación. La metodología empleada y los resultados del proceso de autoevaluación demostraron que el programa cumple con alto nivel de calidad, por lo cual, mediante Resolución 16036 del 10 de diciembre de 2012, el Gobierno, a través del Consejo Nacional de Acreditación, otorga la renovación de acreditación de alta calidad articulando recomendaciones a partir de la evaluación externa y final. Dicho reconocimiento se convierte en un estímulo para el mejoramiento continuo del programa y de cada uno de los actores que lo representan.

PALABRAS CLAVE: autoevaluación, acreditación de alta calidad.

\section{Leonardo Ortegón Cortázar (Colombia) Institución Universitaria Politécnico} Grancolombiano

Coordinador del proceso de autoevaluación y renovación de acreditación del programa de

Mercadeo y Publicidad (2009-2011)

Doctorando en Marketing

lortegon@poligran.edu.co

\section{en}

\section{ABSTRACT}

This article, result of an investigation, aims to register the self-assessment process regarding the renewal of the high quality certification given to the Marketing and Advertising Program of Politécnico Grancolombiano University Institution based on the guidelines established by the National Council of Accreditation. The methodology used as well as the results of the self-assessment process showed that the program meets the high quality level. For this reason, by means of the Resolution 16036, dated December 10th, 2012, the Government, through the National Council of Accreditation, gives the renewal of the high quality certification by putting together recommendations from a final external evaluation. This certification is an incentive to continue improving not only the program, but also the work of each one of its members.

KEYWORDS: Self-assessment, high quality certification

\section{por}

\section{RESUMO}

O presente artigo, resultado de pesquisa, pretende registrar o processo de autoavaliação orientado à renovação da acreditação de alta qualidade do programa de Marketing e Publicidade da Instituição Universitária Politécnico Grancolombiano em concordância com as linhas do Conselho Nacional de Acreditação. A metodologia utilizada e resultados do processo de autoavaliação demostraram que o programa cumpre com alto nível de qualidade, pelo qual, mediante Resolução 16036 de 10 de dezembro de 2012, o Governo, a través do Conselho Nacional de Acreditação outorga a renovação de acreditação de alta qualidade articulando recomendações a partir da avaliação externa e final. Dito reconhecimento se converte em um estímulo para o melhoramento continuo do programa e de cada um dos atores que o representam.

PALAVRAS CHAVE: Autoavaliação, acreditação de alta qualidade.

PARA CITAR ESTE ARTíCUlo/to CITE THIS ARTICle/PARA CITAR ESTE ARTIGO:

Ortegón Cortázar, L. (2014). Autoevaluación y renovación de la acreditación de alta calidad del programa de Mercadeo y Publicidad de la Institución Universitaria Politécnico Grancolombiano. 
El programa de Mercadeo y Publicidad en modalidad presencial, que se ofrece por ciclos propedéuticos por parte de la Institución Universitaria Politécnico Grancolombiano, fue acreditado el 25 de octubre de 2007 por un periodo de cuatro años. Para el programa, este proceso de autoevaluación fue muy significativo dado que pudo ser una excelente oportunidad para analizar su evolución, sus logros y oportunidades de mejoramiento. En este sentido, en 2011 se realizó nuevamente el proceso de autoevaluación para la renovación de la acreditación otorgada por parte del Consejo Nacional de Acreditación (CNA $)^{1}$, logrando nuevamente el reconocimiento de calidad superior por un periodo de cuatro años.

El proceso de renovación de la acreditación de programas por parte del CNA implica desarrollar acciones institucionales y del programa para mantener las fortalezas y solucionar las debilidades autoevaluadas mediante un proceso participativo y transparente, en el que la comunidad universitaria se hace responsable del avance frente a las recomendaciones de la evaluación externa y final de la acreditación otorgada.

La Institución Universitaria Politécnico Grancolombiano expresó la voluntad de renovar la acreditación del programa de Mercadeo y Publicidad en modalidad presencial, y destinó los recursos necesarios para apoyar el proceso de autoevaluación en el plan de desarrollo del programa. La autoevaluación se desarrolló mediante el modelo de autoevaluación para programas diseñado por el CNA con las adaptaciones necesarias a la identidad institucional y la naturaleza propia del programa.

Los pares académicos designados por el CNA para la evaluación externa del programa para renovación de la acreditación fueron Charles Robin Arosa Carrera, coordinador, y Edgar Mauricio López Sarmiento. La evaluación final realizada por el CNA otorgó la renovación de la acreditación mediante Resolución 16036 del 10 de diciembre de 2012.

\footnotetext{
1 La acreditación es un camino para el reconocimiento por parte del Estado colombiano de la calidad de instituciones de educación superior y de programas académicos. Pretende comparar la formación que se imparte con la que reconocen como válida y deseable los pares académicos, es decir, quienes, por poseer las cualidades esenciales de la comunidad académica en un determinado saber, son los representantes del deber ser de es a comunidad. También es un instrumento para promover y reconocer la dinámica del mejoramiento de la calidad y para precisar metas de desarrollo institucional (bttp://www.cna.gov.co/1741/article-186377.html).
}

El proyecto educativo del programa de Mercadeo y Publicidad en coherencia con el Proyecto Educativo Institucional se encuentra enmarcado en su misión institucional:

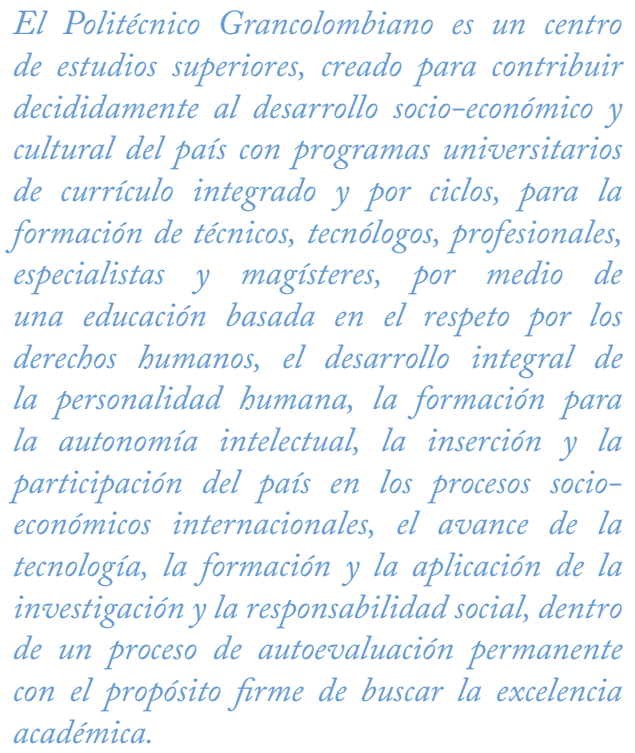

En esta filosofía institucional, el Proyecto Educativo del programa de Mercadeo y Publicidad se describe en los siguientes propósitos:

- Brindar una educación incluyente, bajo el precepto de que la educación enaltece, brinda oportunidades y ayuda a eliminar diferencias socioeconómicas.

- Promover la educación para toda la vida, por medio de la integración entre la educación formal y la educación para el trabajo y el desarrollo personal.

- Fortalecer la autonomía intelectual y la responsabilidad académica y social de la comunidad grancolombiana.

- Promover la flexibilidad y la autonomía brindando a los estudiantes la oportunidad de asumir su educación a través de una cadena de estudios expresada en créditos académicos, que le permita integrarse de forma rápida al mundo laboral y mantener su proceso de formación integral en todos los niveles de la educación formal o de la capacitación para el trabajo y el desarrollo humano. Además desarrollando la capacidad para regirse por sus propias normas con responsabilidad académica para que construyan proyectos de vida felices y exitosos e interactúen integralmente en el mundo actual, con la capacidad de adaptarse a los retos y a los cambios del contexto, de tomar decisiones y de resolver problemas acertadamente.

- Impulsar la formación en investigación para educar ciudadanos autónomos y emprendedores, críticos frente a su realidad y éticamente competentes. y renovación de 
- Aplicar las tecnologías de información y comunicación para permitir una mayor autoformación de los estudiantes, por medio de metodologías presenciales, semipresenciales y virtuales.

- Fomentar la educación superior, con costos accesibles para la mayoría de los colombianos.

- Velar por mantener altos estándares de calidad académica en todos sus programas académicos.

- Adquirir competencias integrales para saber ser, saber aprender, saber hacer y saber emprender, en contexto.

Para la difusión de estos propósitos entre la comunidad académica, se cuenta con el apoyo del Departamento de Comunicación Institucional y con la agencia de publicidad Trompo que utilizan los canales de comunicación institucionales, tales como correo institucional, página web $^{2}$, carteleras físicas, Moodle, Centro de Información de la Organización, pantallas de televisión GEN, redes sociales, blogs oficiales, boletines y newsletter institucionales. El programa cuenta además con otros medios como la emisora Poliradio, Polimedios (página de difusión de proyectos académicos) y espacios de inducción a estudiantes y docentes donde se realiza una presentación formal de la Facultad y del programa y también se entrega material promocional en formato multimedia.

El programa de Mercadeo y Publicidad de la Institución Universitaria Politécnico Grancolombiano pertenece a la Facultad de Mercadeo, Comunicación y Artes, y expresa el objeto social de su labor en su proyecto de formar profesionales integrales para los procesos de investigación, planeación, administración y evaluación del mercadeo y la publicidad, para que con su conocimiento aporten valor a todos los integrantes de la cadena de producción-consumo. Este propósito contribuye al cumplimiento de la misión institucional mediante la formación, por ciclos, de tecnólogos en Mercadeo y $\mathrm{Pu}-$ blicidad y de profesionales en Mercadeo y Publicidad.

Panorama l

Para desarrollar en el programa académico las funciones de docencia, investigación, internacionalización, extensión o proyección social y bienestar de la comunidad institucional, se dispone en lo institucional de procesos de planeación estratégica que brindan los elementos que soportan la toma de decisiones. Al respecto, la Institución implementó desde 2009 la metodología Balanced ScoreCard, que interrelaciona los elementos esenciales del sistema, sintetizando visual y gráficamente la dinámica de la Institución, por medio de relaciones causaefecto, da prioridad a los elementos en los que se debe focalizar la gestión estratégica e involucra a todos los colaboradores de la organización. En la actualidad, la Institución se mueve alrededor de su mapa estratégico y realiza sesiones periódicas de seguimiento de cada estrategia, denominadas reuniones de análisis estratégico. Todas enmarcadas en la propuesta de valor institucional que contempla la formación integral, para la autonomía y la competitividad, excelencia y pertinencia de los programas, inclusión, flexibilidad, excelencia en el servicio y precio justo.

En concordancia con la misión y la visión institucional, el programa de Mercadeo y Publicidad ha definido su proyecto educativo a partir de los siguientes objetivos de formación:

El objetivo general del programa es formar profesionales integrales para los procesos de investigación, planeación, administración y evaluación del mercadeo y la publicidad, a fin de que con su conocimiento aporten valor a todos los integrantes de la cadena de producción/consumo.

El primer ciclo tiene por objetivo formar tecnólogos en mercadeo y publicidad capaces de desarrollar, planear, dirigir, aplicar y evaluar los procesos del marketing y de la publicidad, sustentados en el conocimiento de los principios teórico prácticos, que dan sustento científico y tecnológico a las dos disciplinas. Además de lo anterior, la opción propedéutica prepara al estudiante en los aspectos administrativos, financieros y comunicacionales que permiten generar propuestas estratégicas y creativas más integrales y pertinentes desde enfoques tanto económicos, administrativos como sociales. En el segundo ciclo, el objetivo es formar profesionales capaces de integrar y apropiar todos los procesos creativos, estratégicos, administrativos y gerenciales en mercadeo y publicidad, adaptando la teoría y procedimientos conocidos a las realidades propias del contexto en el que se desempeñan.

En complemento, el programa cuenta con diferentes escenarios para el seguimiento, difusión y actualización tanto del desarrollo académico como del cumplimiento de los lineamientos estratégicos del programa. Estos espacios son los siguientes: 
- Consejo de Facultad: estudia y aprueba las diferentes propuestas sobre cambios fundamentales en el currículo, está liderado por el decano, con la participación del representante de egresados, representante de estudiantes, representante de profesores, directores académicos de departamento y coordinadores de área.

- Comité Académico de Facultad: órgano integrador de todos los estamentos académicos de la Facultad y regido por el decano, su objetivo es propiciar espacios de diálogo para la reflexión y toma de decisiones académicas de la Facultad.

- Comité de Departamento Académico: reúne y estudia las propuestas de cada una de las áreas del programa para determinar el camino de acuerdo con la pertinencia y claridad de las propuestas presentadas. Estas decisiones son llevadas al Consejo Académico de Facultad.

- Comités curriculares de área: revisan, evalúan y proponen innovaciones, cambios y modificaciones al plan de estudio en el área correspondiente. Al respecto, en 2004, como parte del proceso de mejoramiento continuo de la calidad académica, la entonces Facultad de Mercadeo y Publicidad realizó una reforma curricular en la que intervinieron sus profesores y directivos de planta, acompañados de expertos temáticos en las áreas de mercadeo y de publicidad, que en esencia permitió pasar del enfoque de mercadeo masivo al de mercadeo relacional, y complementar la publicidad como factor de mercadeo con los componentes fundamentales de la construcción de marca. Para 2007, se realizó una reforma al plan académico, que buscó una mayor flexibilidad conservando los principios académicos de interdisciplinariedad, definido por la generación de valor como concepto, que correlaciona la profesión y el oficio, y por los hilos conductores de la comunicación, la estrategia, la creatividad, la responsabilidad y el uso adecuado de la tecnología.

El plan de estudios se estructura con asignaturas de fundamentación, de práctica y de aplicación: las primeras corresponden a la base epistemológica de las ciencias que le dan contexto teórico-práctico al mercadeo y la publicidad; las segundas son aquellas en las que el estudiante aplica los procesos, procedimientos, principios y teorías derivados de las asignaturas de fundamentación, $\mathrm{y}$ las terceras corresponden a aquellas asignaturas en las que se pueden generar prácticas con objetos de estudios reales, tales como la investigación de mercados, el plan de mercadeo, las campañas publicitarias y las gerencias de mercadeo I y II del ciclo profesional, entre otras, lo que les permite hacer aportes al contexto. Por otra parte, el programa también busca articular la participación de profesores y estudiantes en actividades de cooperación académica logrando el desarrollo de proyectos específicos. Así es como a través de las diferentes líneas de los grupos de investigación se han obtenido experiencias de visibilidad e impacto académico.

Todos los anteriores cambios y la actualización del plan curricular están soportados por la investigación, la asistencia a eventos gremiales y académicos, los resultados de evaluaciones de las prácticas laborales de los estudiantes, la representación como ponentes a nombre de la Facultad, la revisión de las tendencias internacionales en el área de conocimiento y la producción intelectual por medio de textos y documentos publicados por revistas indexadas en el ámbito nacional e internacional. Estos mecanismos hacen que continuamente se revisen las tendencias de la disciplina, los programas académicos nacionales e internacionales y se reciba una retroalimentación del sector empresarial y de los egresados. En este sentido, es de destacar que el programa profesional de Mercadeo y Publicidad fue el primer programa de educación superior en el país que integró las dos disciplinas, el mercadeo y la publicidad.

\section{EXPERIENCIAS SIGNIFICATIVAS DEL PROGRAMA DURANTE LA ACREDITACIÓN}

Durante el tiempo de vigencia de la acreditación, se evidencia el fortalecimiento de la cultura de la autoevaluación y el mejoramiento de la vida universitaria. Las principales experiencias son:

- Organización del plan de estudios por bloques y adopción del sistema de créditos académicos, permitiendo una mayor sinergia con otros programas académicos y una mayor autonomía del estudiante en su proceso formativo.

- Creación del programa de Permanencia en el Poli, que ha buscado mejorar la atención de los estudiantes, fue seleccionado como una buena práctica en la primera convocatoria de reconocimiento y publicación de buenas prácticas que organizó Telescopi (Colombia) (Red de Observatorios de Buenas Prácticas de Dirección Estratégica Universitaria en Latinoamérica y Europa).

- Clasificación en categoría D del escalafón de Colciencias de dos grupos de investigación: Mercadeo I+2 y Comunicación Estratégica y Creativa de la Institución Universitaria Politécnico Grancolombiano.

- Aumento de artículos publicados en revistas indexadas y no indexadas: nacionales e internacionales.

- Incremento en la participación en eventos y congresos nacionales e internacionales.
Autoevaluación

y renovación de la acreditación de alta calidad del programa de Mercadeo y Publicidad de la Institución Universitaria Politécnico Grancolombiano

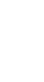


- Vinculación de toda la planta docente de la Facultad con contrato laboral a término indefinido; no hay docentes de medio tiempo ni con contratos a término fijo.

- Disminución del índice de deserción a partir del programa Permanencia en el Poli.

- Vinculación del programa con otras organizaciones y redes de formación de reconocido prestigio nacional e internacional.

- Organización del Primer Encuentro Latinoamericano de Facultades y Escuelas de Mercadeo y Publicidad de América Latina.

- Participación en el grupo de instituciones fundadoras de la Asociación de Programas de Mercadeo.

- Miembro del Consejo Editorial de la Revista Publicidad y Mercadeo.

- Miembro de la Red Latinoamericana de Cooperación Universitaria.

- Miembro de la Internacional Advertising Association, entidad que certifica programas académicos en este campo y de la cual se ha recibido la acreditación internacional en marketing y comunicaciones en 1998, ratificada en 2004.

- Mejoramiento del clima laboral en la Facultad con ejecución del programa ¿Cómo Vamos?

- Mejora en la titulación de los profesores (incremento en el número de profesores con posgrado).

- Buen posicionamiento de los egresados en el mercado laboral.

\section{Autoevaluación}

El proceso de autoevaluación para la renovación de la acreditación del programa atendió los lineamientos emitidos por el CNA. El modelo aplicado fue el vigente propuesto por el CNA (2006) en el documento Lineamientos para la acreditación de programas.

\section{PONDERACIÓN}

Siguiendo los Lineamientos del CNA, el ejercicio tuvo como propósito establecer los pesos (porcentajes) que se asignarían a cada factor y característica para realizar la autoevaluación de la calidad del programa. Debe advertirse que existen dos tipos de ponderación de los factores: uno de carácter institucional (tabla 1) y otro de las características de los factores realizado por el programa de Mercadeo y Publicidad de acuerdo con su naturaleza y particularidades. El primero fue efectuado por el cuerpo directivo y el segundo por un comité ad hoc integrado
TABLA 1. PONDERACIÓN DE FACTORES DE AUTOEVALUACIÓN DE 2006 Y 2011

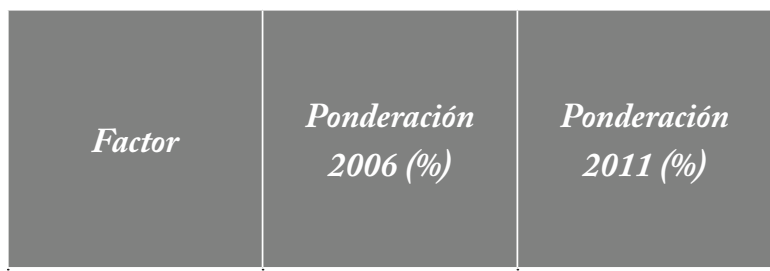

\begin{tabular}{|c|c|c|}
\hline $\begin{array}{l}\text { Procesos } \\
\text { académicos }\end{array}$ & 19 & 12 \\
\hline $\begin{array}{l}\text { Misión y } \\
\text { proyecto } \\
\text { institucional }\end{array}$ & 15 & 13 \\
\hline Estudiantes & 12 & 13 \\
\hline Profesores & 12 & 17 \\
\hline $\begin{array}{l}\text { Organización, } \\
\text { administración } \\
\text { y gestión }\end{array}$ & 11 & 12 \\
\hline $\begin{array}{l}\text { Recursos físicos } \\
\text { y financieros }\end{array}$ & 11 & 10 \\
\hline $\begin{array}{l}\text { Bienestar } \\
\text { institucional }\end{array}$ & 11 & 13 \\
\hline $\begin{array}{l}\text { Egresados e } \\
\text { impacto sobre el } \\
\text { medio }\end{array}$ & 9 & 10 \\
\hline
\end{tabular}

Metodología

La población participante estuvo conformada por estudiantes, docentes, directivos, empleados, egresados y empleadores. Se diseñaron diversos instrumentos con preguntas orientadas a identificar las percepciones y niveles de acuerdo con ítems formulados en forma positiva y para el registro individual de aspectos por mejorar. En los talleres realizados con estudiantes, docentes y egresados, se registraron los acuerdos sobre el estado de cumplimiento de las características por factor del modelo de acreditación para programas de pregrado, el estado de avance sobre las recomendaciones señaladas por el CNA en el anterior proceso y las acciones de la Facultad y el programa para mantener las fortalezas, reorientar acciones o solucionar debilidades. 
A partir de la ponderación, se procedió a calificar el grado de cumplimiento de cada característica, se utilizó la misma escala aplicada en la autoevaluación de 2006. De la combinación de la calificación de cada característica con la calificación de su grado de cumplimiento, se obtuvo la valoración de las características y del factor al que pertenecen. La tabla 2 registra el grado de cumplimiento de cada característica en la autoevaluación 2011 y su relación con el resultado de la autoevaluación realizada en 2006.

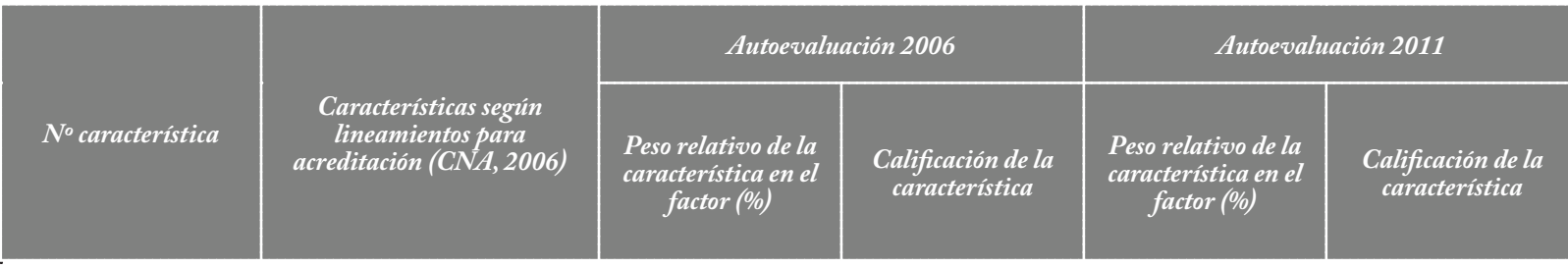

\section{MISIÓN PROYECTO INSTITUCIONAL}

\begin{tabular}{|c|c|c|c|c|c|}
\hline 1 & Misión institucional & 25 & 4.6 & 28 & 4.0 \\
\hline 2 & Proyecto institucional & 25 & 4.6 & 28 & 4.4 \\
\hline 3 & $\begin{array}{l}\text { Proyecto educativo del } \\
\text { programa }\end{array}$ & 25 & 4.5 & 23 & 4.1 \\
\hline 4 & $\begin{array}{l}\text { Relevancia académica } \\
\text { y pertinencia social del } \\
\text { programa }\end{array}$ & 25 & 4.5 & 21 & 4.5 \\
\hline \multicolumn{6}{|c|}{ ESTUDIANTES } \\
\hline 5 & $\begin{array}{l}\text { Mecanismos de } \\
\text { ingreso }\end{array}$ & 21 & 4.4 & 16 & 4.4 \\
\hline 6 & $\begin{array}{l}\text { Número y calidad } \\
\text { de los estudiantes } \\
\text { admitidos }\end{array}$ & 21 & 4.0 & 17 & 4.4 \\
\hline
\end{tabular}




\begin{tabular}{|c|c|c|c|c|c|c|}
\hline \multirow[b]{2}{*}{ Leonardo } & \multirow{3}{*}{ No característica } & \multirow{3}{*}{$\begin{array}{l}\text { Caracteristicas seguin } \\
\text { lineamientos para } \\
\text { acreditación (CNA, 2006) }\end{array}$} & \multicolumn{2}{|c|}{ Autoevaluación 2006} & \multicolumn{2}{|c|}{ Autoevaluación 2011} \\
\hline & & & $\begin{array}{l}\text { Peso relativo de la } \\
\text { característica en el } \\
\text { factor (\%) }\end{array}$ & $\begin{array}{l}\text { Calificación de la } \\
\text { característica }\end{array}$ & $\begin{array}{l}\text { Peso relativo de la } \\
\text { caracteristica en el } \\
\text { factor (\%) }\end{array}$ & $\begin{array}{l}\text { Calificación de la } \\
\text { característica }\end{array}$ \\
\hline Ortegón & & & & & & \\
\hline \multirow[t]{10}{*}{ Cortázar I } & 7 & $\begin{array}{l}\text { Permanencia y } \\
\text { deserción estudiantil }\end{array}$ & 19 & 4.2 & 22 & 4.6 \\
\hline & 8 & $\begin{array}{l}\text { Participación en } \\
\text { actividades de } \\
\text { formación integral }\end{array}$ & 19 & 4.3 & 23 & 4.3 \\
\hline & 9 & $\begin{array}{l}\text { Reglamento } \\
\text { estudiantil }\end{array}$ & 21 & 4.6 & 22 & 4.2 \\
\hline & \multicolumn{6}{|c|}{ PROFESORES } \\
\hline & 10 & $\begin{array}{l}\text { Selección y vinculación } \\
\text { de profesores }\end{array}$ & 16 & 4.3 & 19 & 4.2 \\
\hline & 11 & Estatuto profesoral & 8 & 4.2 & 13 & 4.1 \\
\hline & 12 & $\begin{array}{l}\text { Número, dedicación y } \\
\text { nivel de formación de } \\
\text { los profesores }\end{array}$ & 16 & .0 & 17 & 4.3 \\
\hline & 13 & Desarrollo profesoral & 16 & 4.1 & 14 & 4.4 \\
\hline & 14 & $\begin{array}{l}\text { Interacción con } \\
\text { las comunidades } \\
\text { académicas }\end{array}$ & 12 & 3.7 & 8 & 4.4 \\
\hline & 15 & $\begin{array}{l}\text { Estímulos a } \\
\text { la docencia, } \\
\text { investigación, } \\
\text { extensión o } \\
\text { proyección social } \\
\text { y a la cooperación } \\
\text { internacional }\end{array}$ & 12 & 4.0 & 10 & 4.4 \\
\hline $\begin{array}{l}\text { Volumen } 8 \text { I } \\
\text { Número } 14 \text { I } \\
\text { Enero - Junio I }\end{array}$ & 16 & $\begin{array}{l}\text { Producción de } \\
\text { material docente }\end{array}$ & 8 & 4.0 & 8 & 4.1 \\
\hline & 17 & $\begin{array}{l}\text { Remuneración por } \\
\text { méritos }\end{array}$ & 12 & 3.8 & 11 & 3.9 \\
\hline
\end{tabular}



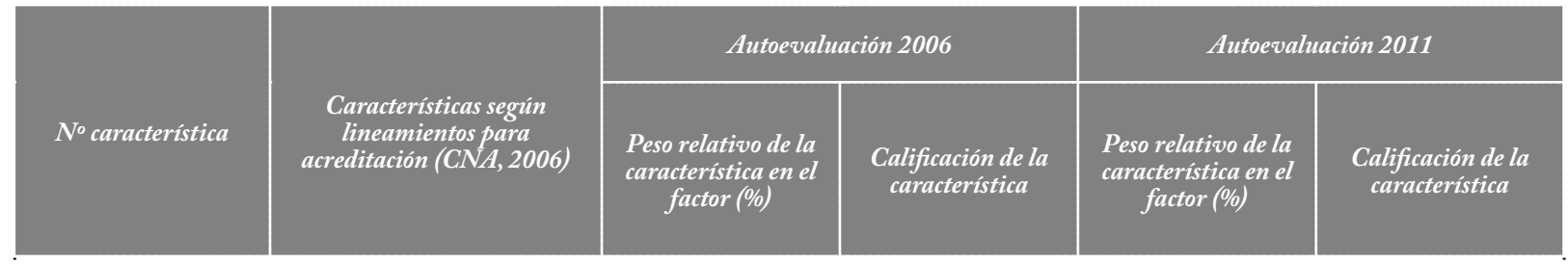

Autoevaluación y renovación de la acreditación de alta calidad del programa de Mercadeo y

\section{PROCESOS CADÉMICOS}

\section{Publicidad de}

la Institución

Universitaria

\begin{tabular}{|c|c|c|c|c|c|}
\hline 18 & $\begin{array}{l}\text { Integralidad del } \\
\text { currículo }\end{array}$ & 9 & 4.5 & 9 & 4.4 \\
\hline 19 & $\begin{array}{l}\text { Flexibilidad del } \\
\text { currículo }\end{array}$ & 9 & 4.5 & 6 & 4.3 \\
\hline 20 & Interdisciplinariedad & 9 & 3.4 & 5 & 4.3 \\
\hline 21 & $\begin{array}{l}\text { Relaciones nacionales } \\
\text { e internacionales del } \\
\text { programa }\end{array}$ & 9 & 4.5 & 9 & 4.7 \\
\hline 22 & $\begin{array}{l}\text { Metodologías } \\
\text { de enseñanza y } \\
\text { aprendizaje }\end{array}$ & 6 & 4.5 & 11 & 4.4 \\
\hline 23 & $\begin{array}{l}\text { Sistema de evaluación } \\
\text { de estudiantes }\end{array}$ & 6 & 4.3 & 8 & 4.1 \\
\hline 24 & $\begin{array}{l}\text { Trabajos de los } \\
\text { estudiantes }\end{array}$ & 6 & 4.2 & 8 & 4.4 \\
\hline 25 & $\begin{array}{l}\text { Evaluación y } \\
\text { autorregulación del } \\
\text { programa }\end{array}$ & 6 & 4.4 & 9 & 4.0 \\
\hline 26 & $\begin{array}{l}\text { Formación para la } \\
\text { investigación }\end{array}$ & 9 & $4 ., 4$ & 6 & 4.4 \\
\hline 27 & $\begin{array}{l}\text { Compromiso con la } \\
\text { investigación }\end{array}$ & 6 & 4.0 & 6 & 4.3 \\
\hline 28 & $\begin{array}{l}\text { Extensión o } \\
\text { proyección social }\end{array}$ & 9 & 4.3 & 6 & 4.1 \\
\hline
\end{tabular}




\begin{tabular}{|c|c|c|c|c|c|c|}
\hline \multirow[b]{2}{*}{ Leonardo } & \multirow[b]{2}{*}{ No característica } & \multirow[b]{2}{*}{$\begin{array}{l}\text { Caracteristicas según } \\
\text { lineamientos para } \\
\text { acreditación }(C N A, 2006)\end{array}$} & \multicolumn{2}{|c|}{ Autoevaluación 2006} & \multicolumn{2}{|c|}{ Autoevaluación 2011} \\
\hline & & & $\begin{array}{l}\text { Peso relativo de la } \\
\text { caracteristica en el } \\
\quad \text { factor (\%) }\end{array}$ & $\begin{array}{l}\text { Calificación de la } \\
\text { característica }\end{array}$ & $\begin{array}{l}\text { Peso relativo de la } \\
\text { característica en el } \\
\quad \text { factor (\%) }\end{array}$ & $\begin{array}{l}\text { Calificación de la } \\
\text { caracteristica }\end{array}$ \\
\hline Ortegón & & & & & & \\
\hline \multirow[t]{11}{*}{ Cortázar I } & & & & & & \\
\hline & 29 & Recursos bibliográficos & 6 & 4.3 & 7 & 4.3 \\
\hline & 30 & $\begin{array}{l}\text { Recursos informáticos } \\
\text { y de comunicación }\end{array}$ & 6 & 3.8 & 6 & 4.4 \\
\hline & 31 & $\begin{array}{l}\text { Recursos de apoyo } \\
\text { docente }\end{array}$ & 5 & 3.8 & 6 & 4.6 \\
\hline & \multicolumn{6}{|c|}{ BIENESTAR INSTITUCIONAL } \\
\hline & 32 & $\begin{array}{l}\text { Políticas, programas y } \\
\text { servicios de bienestar } \\
\text { universitario }\end{array}$ & 100 & 4.4 & 100 & 4.3 \\
\hline & \multicolumn{6}{|c|}{ ORGANIZACIÓN ADMINISTRACIÓN Y GESTIÓN } \\
\hline & 33 & $\begin{array}{l}\text { Organización, } \\
\text { administración y } \\
\text { gestión del programa }\end{array}$ & 35 & 4.5 & 29 & 4.2 \\
\hline & 34 & $\begin{array}{l}\text { Sistemas de } \\
\text { comunicación e } \\
\text { información }\end{array}$ & 20 & 4.0 & 22 & 4.0 \\
\hline & 35 & $\begin{array}{l}\text { Dirección del } \\
\text { programa }\end{array}$ & 30 & 4.4 & 30 & 4.3 \\
\hline & 36 & $\begin{array}{l}\text { Promoción del } \\
\text { programa }\end{array}$ & 15 & 4.5 & 19 & 4.3 \\
\hline \multicolumn{7}{|l|}{ Panorama I } \\
\hline $\begin{array}{l}\text { pp. 73-86 | } \\
\text { Volumen } 81\end{array}$ & \multirow{2}{*}{\multicolumn{6}{|c|}{ EGRESADOS E IMPACTO SOBRE EL MEDIO }} \\
\hline Número 141 & & & & & & \\
\hline Enero - Junio I & & & & & & \\
\hline $2014 \mid$ & 37 & $\begin{array}{l}\text { Influencia del } \\
\text { programa en el medio }\end{array}$ & 35 & 4.2 & 35 & 4.4 \\
\hline
\end{tabular}




\begin{tabular}{|c|c|c|c|c|c|}
\hline \multirow[b]{2}{*}{$N^{o}$ característica } & \multirow[b]{2}{*}{$\begin{array}{c}\text { Características seguin } \\
\text { lineamientos para } \\
\text { acreditación }(C N A, 2006)\end{array}$} & \multicolumn{2}{|c|}{ Autoevaluación 2006} & \multicolumn{2}{|c|}{ Autoevaluación 2011} \\
\hline & & $\begin{array}{l}\text { Peso relativo de la } \\
\text { caracteristica en el } \\
\quad \text { factor (\%) }\end{array}$ & $\begin{array}{l}\text { Calificación de la } \\
\text { característica }\end{array}$ & $\begin{array}{l}\text { Peso relativo de la } \\
\text { caracteristica en el } \\
\quad \text { factor (\%) }\end{array}$ & $\begin{array}{l}\text { Calificación de la } \\
\text { característica }\end{array}$ \\
\hline 38 & $\begin{array}{l}\text { Seguimiento de los } \\
\text { egresados }\end{array}$ & 28 & 3,9 & 27 & 4.0 \\
\hline 39 & $\begin{array}{l}\text { Impacto de los } \\
\text { egresados en el medio } \\
\text { social y académico }\end{array}$ & 37 & 3.8 & 38 & 4.5 \\
\hline \multicolumn{6}{|c|}{ RECURSOS FÍSICOS Y FINANCIEROS } \\
\hline 40 & Recursos físicos & 35 & 4.1 & 32 & 4.0 \\
\hline 41 & $\begin{array}{l}\text { Presupuesto del } \\
\text { programa }\end{array}$ & 35 & 4.4 & 40 & 4.4 \\
\hline 42 & $\begin{array}{l}\text { Administración de los } \\
\text { recursos }\end{array}$ & 30 & 4.4 & 28 & 4.6 \\
\hline
\end{tabular}

En la tabla 3 se registra el resultado del juicio de calidad de la autoevaluación realizada en 2006 y 2011, obtenida de la calificación de los factores y el aporte a la calificación total del programa. Para la realización de la calificación, se siguieron dos etapas. En una primera, cada miembro del comité ad hoc realizó un ejercicio previo de calificación individual teniendo como base el informe autoevaluativo y las fortalezas y debilidades identificadas por la comunidad académica. Los resultados de la calificación inicial realizada por cada miembro y su justificación fueron consolidados y compartidos por los miembros del comité, y tenidos en cuenta durante las deliberaciones y por consenso se obtuvo la calificación de cada una de las características de alta calidad. Finalmente, la calificación de cada factor resultó de la ponderación de la sumatoria de las calificaciones de las características teniendo en cuenta el respectivo peso de cada factor y la calificación final se obtuvo tomando como base la sumatoria de todos los factores. 


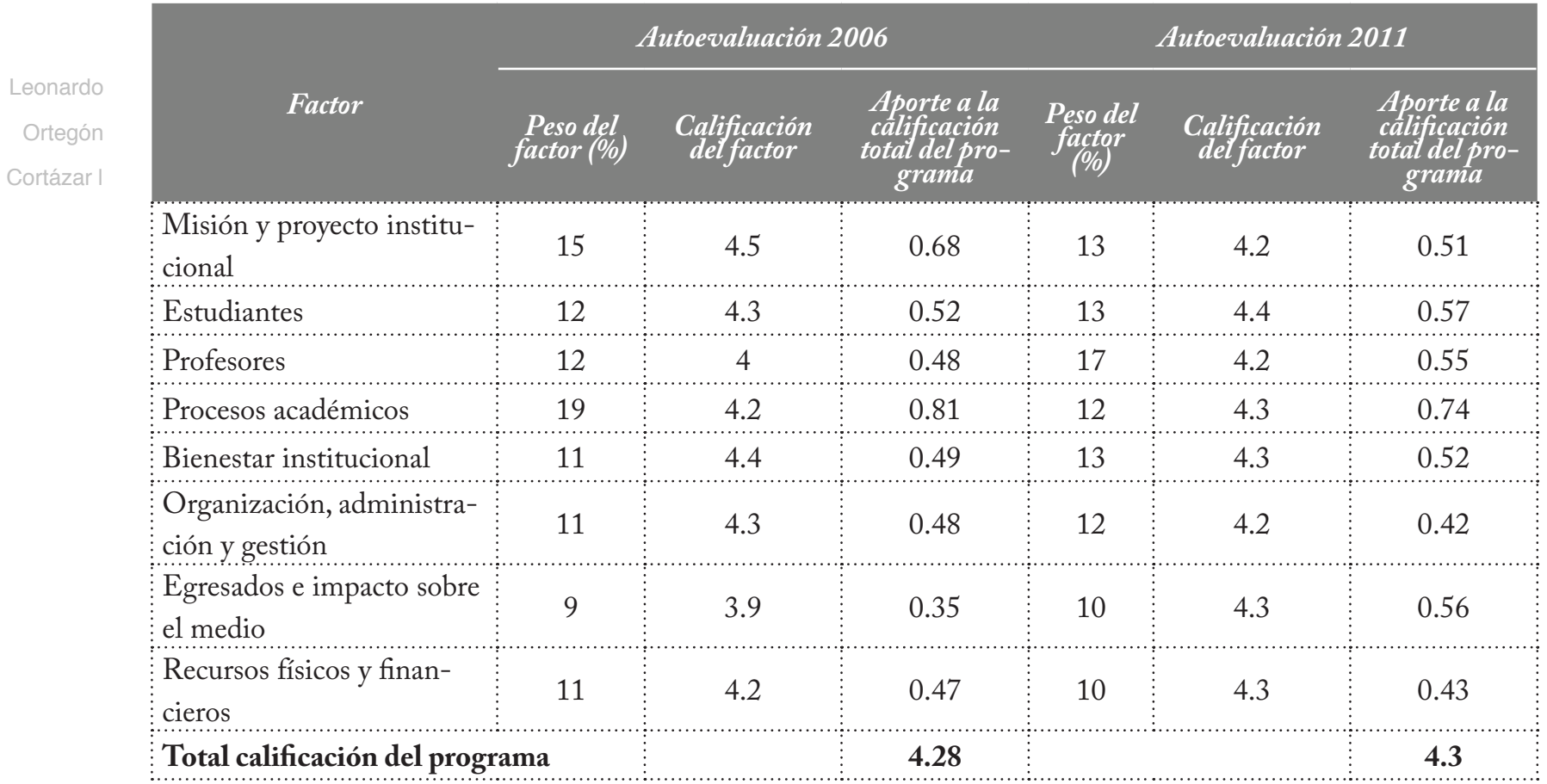

Panorama I pp. 73-86। Volumen 81 Número 14 I
De acuerdo con la escala de calificación adoptada, el programa obtuvo una calificación global de 4.30 que la ubica en el rango de alto grado de calidad. Una comparación entre las calificaciones obtenidas en los dos procesos de autoevaluación permite constatar que el programa de Mercadeo y Publicidad continúa realizando su proyecto educativo con calidad.

En contraste, la visita de evaluación externa se cumplió durante los días 12, 13 y 14 de abril de 2012. Se constituyó en un escenario propicio para el fortalecimiento de la cultura de autoevaluación en el que estudiantes, docentes, egresados y directivos en diálogo académico demostraron el sentido de pertenencia y de compromiso con el mejoramiento del programa y de la institución.

\section{EVALUACIÓN}

El CNA registró las siguientes observaciones del programa, por las cuales considera que tiene alta calidad, y que hacen parte de la resolución de renovación de la acreditación del programa:

- El compromiso del programa con el mejoramiento continuo, expresado en el cumplimiento de la gran mayoría de requerimientos que se dedujeron del pasado proceso de autoevaluación y acreditación.

- Estudiantes y docentes identificados con la misión de la universidad y con el proyecto educativo del programa, con alto sentido de pertenencia y compromiso.

- El mecanismo de ingreso de los estudiantes, amplio y bien divulgado, consistente en pruebas y entrevistas.

- Los apoyos dados a los estudiantes como orientación para préstamos para matrículas, becas por calidad académica, además de la asesoría académica brindada por los profesores del programa y los servicios de bienestar institucional.

- El Departamento de Investigación, Desarrollo e Innovación, recientemente estructurado, generando convocatorias para el desarrollo de proyectos de investigación y fomentando las publicaciones de sus resultados.

- La presencia de los egresados en actividades y vida académica de la Facultad y su impacto a nivel local y regional, reconocido por los empleadores.

Igualmente, el CNA registró las siguientes recomendaciones para fortalecer y contribuir con el adecuado desarrollo del programa:

- Intensificar los esfuerzos para aumentar el número de profesores de planta, a expensas de los de cátedra, 
permitiendo un mejor desarrollo de las actividades misionales del programa.

- Continuar con el proceso de cualificación de los docentes a niveles de maestría y doctorado.

- Fomentar las publicaciones de los docentes en revistas indexadas nacionales e internacionales.

- Dar mayor apoyo a los grupos de investigación del programa, para que logren mejores categorías en el Sistema Nacional de Ciencia y Tecnología.

- Ampliar la divulgación del reglamento docente y contemplar la posibilidad de mejorar los espacios físicos de los docentes.

- Fortalecer las habilidades de estudiantes y profesores en una segunda lengua.

- Hacer un mejor seguimiento y supervisión a las prácticas empresariales de los estudiantes.

- Diseñar estrategias para mejorar la base de datos de los egresados y propiciar su participación activa en los procesos de revisión curricular.

- Continuar buscando mecanismos para reducir la deserción estudiantil.

\section{PROPUESTAS DE MEJORAMIENTO}

Según el proceso de autoevaluación realizado, el programa y la Facultad alinearon los resultados a las propuestas y planes de mejoramiento de la institución para asegurar su implementación. Dichas propuestas se traducen en acciones de mejoramiento definidas por la Facultad siendo categorizadas en seis dimensiones de funcionamiento (comunicación e identidad institucional, pertinencia del plan de estudios, investigación y extensión social del programa, fortalecimiento del cuerpo docente, seguimiento a egresados y mejoramiento de la infraestructura). Dichos propósitos se expresan en los siguientes objetivos por cumplir en este periodo de alta calidad académica otorgado por el CNA, desde 2013 hasta 2016.

- Garantizar que las decisiones académicas y proyectos que se desarrollan sean conocidos por profesores y estudiantes del programa.

- Apropiar a los profesores y estudiantes del programa de la identidad institucional.

- Examinar de manera periódica las necesidades del entorno y su incidencia en la organización del plan de estudios y fortalecer los sistemas de atención y evaluación de estudiantes.

- Fortalecer e incentivar en la Facultad la función investigativa.
- Fortalecer e incentivar en la Facultad proyectos de extensión y proyección social.

- Fortalecer la calidad del cuerpo profesoral.

- Fortalecer los procesos de seguimiento y vinculación con los egresados.

- Mejorar las condiciones de áreas académicas para profesores y estudiantes de la institución.

Finalmente, el compromiso de los directivos, docentes, estudiantes y egresados permiten mantener y proyectar las fortalezas institucionales y del programa de Mercadeo y Publicidad, superando las debilidades y alcanzando los niveles de alta calidad deseados.

\section{REFERENCIAS BIBLIOGRÁFICAS}

1. Colombia, Consejo Nacional de Acreditación (2006). Guía de procedimiento. Autoevaluación con fines de acreditación de programas de pregrado (4a ed.). Recuperado de http://cms-static. colombiaaprende.edu.co/cache/binaries/articles-186376_auto_eval_prog_aca_pregrado. pdf?binary_rand $=6566$

2. Colombia, Consejo Nacional de Acreditación (2006). Guía de procedimiento. Renovación de la acreditación de programas académicos de pregrado. Recuperado de http://cms-static. colombiaaprende.edu.co/cache/binaries/articles-186376_guia_5.pdf?binary_rand $=1730$

3. Institución Universitaria Politécnico Grancolombiano (2011). Proyecto Educativo Institucional. Recuperado de http://www. poligran.edu.co/comunica/documentosportal/ pei.pdf

4. Institución Universitaria Politécnico Grancolombiano, Facultad de Mercadeo, Comunicación y Artes (s. f.). Planes de mejoramiento y procesos académicos.

5. Institución Universitaria Politécnico Grancolombiano, Facultad de Mercadeo, Comunicación y Artes (2006). Informe de autoevaluación para el registro de acreditación. Bogotá.

6. Institución Universitaria Politécnico Grancolombiano y Facultad de Mercadeo, Comunicación y Artes (2011). Informe de autoevaluación para la renovación de acreditación. Bogotá.

7. Institución Universitaria Politécnico Grancolombiano (2013-2017). Mapa estratégico Institucional. Recuperado de http://cio/PEPG/ Documentos\%20RAE/
Autoevaluación y renovación de la acreditación de alta calidad del programa de Mercadeo y Publicidad de la Institución Universitaria Politécnico 